\title{
Perfil epidemiológico de suicídios notificados no estado do Pará no ano de 2019
}

\author{
Epidemiological profile of suicides reported in the state of Pará in the year 2019 \\ Perfil epidemiológico de los suicidios registrados en el estado de Pará en 2019
}

Recebido: 02/12/2021 | Revisado: 07/12/2021 | Aceito: 08/12/2021 | Publicado: 17/12/2021

\author{
Adria Leitão Maia \\ ORCID: https://orcid.org/0000-0002-4173-1374 \\ Centro Universitário da Amazônia, Brasil \\ E-mail: Adria-maia@hotmail.com \\ Brenda Tanielle Dutra Barros \\ ORCID: https://orcid.org/0000-0002-3184-050X \\ Centro Universitário Metropolitano da Amazônia, Brasil \\ E-mail: Brendatanielle.enf@gmail.com \\ Clívia Naiara Pinto Monteiro \\ ORCID: https://orcid.org/0000-0003-4611-8410 \\ Centro Universitário da Amazônia, Brasil \\ E-mail: Clivia89@gmail.com \\ Rômulo Leno Miranda Barros \\ ORCID: https://orcid.org/0000-0003-1802-4521 \\ Centro Universitário da Amazônia, Brasil \\ E-mail: romuloleno@hotmail.com \\ Taíla Cristina Paiva da Costa \\ ORCID: https://orcid.org/0000-0003-0223-7813 \\ Centro Universitário da Amazônia, Brasil \\ E-mail: taylacosta-14@hotmail.com
}

\begin{abstract}
Resumo
Introdução: A ideação suicida é um elemento fundamental de um processo chamado comportamento suicida, o qual inclui: planejamento, tentativa e o suicídio propriamente dito. Trata-se de um percurso que vai desde a motivação e ideação suicida até o planejamento do método e a autoagressão. O Brasil ocupa a $11^{\mathrm{a}}$ posição no ranking mundial de suicídios. Objetivo: identificar perfil epidemiológico de suicídios notificados no estado do Pará no ano de 2019. Materiais e Métodos: Estudo epidemiológico descritivo, a partir de dados de Suicídio ocorridos no Estado do Pará no ano de 2019, registrados no Sistema de Informações de Mortalidade e disponibilizados pelo Departamento de Informática do Sistema Único de Saúde. Resultados: No ano de 2019 foram notificados no Estado do Pará 348 suicídios, sendo os municípios de Belém (55 casos), Santarém (18 casos) e Itaituba (18 casos), as cidades com maior ocorrência; quanto a variável gênero, verificou-se prevalência do sexo masculino, com $272(78,16 \%)$; relativo à faixa etária, 88 casos $(25,3 \%)$ estavam entre 20 e 29 anos de idade, seguido pela faixa etária compreendida entre 30 e 39 anos apresentando 73 casos (21\%). Conclusão: Constatou-se que apesar de ser bem evidenciado a importância do enfermeiro nessa temática, bem como os elevados índices de suicídios ocorridos no Brasil em foco no estado do Pará, ainda é baixo o número de pesquisas que descrevem a relação entre o profissional de enfermagem frente ao comportamento suicida. Palavras-chave: Suicídio; Saúde mental; Enfermagem psiquiátrica.
\end{abstract}

\begin{abstract}
Introduction: Suicidal ideation is a key element of a process called suicidal behavior, which includes: planning, attempting, and the actual suicide. It is a path that goes from motivation and suicidal ideation to planning the method and self-aggression. Brazil ranks 11th in the world for suicides. Objective: to identify epidemiological profile of suicides reported in the state of Pará in the year 2019. Materials and Methods: Descriptive epidemiological study, based on data of Suicide occurred in the State of Pará in the year 2019, registered in the Mortality Information System and made available by the Department of Informatics of the Unified Health System Results: In the year 2019, 348 suicides were reported in the State of Pará, with the municipalities of Belém (55 cases), Santarém (18 cases) and Itaituba (18 cases), the cities with the highest occurrence; as for the gender variable, there was a prevalence of male gender, with $272(78.16 \%)$; regarding the age range, 88 cases $(25.3 \%)$ were between 20 and 29 years old, followed by the age range between 30 and 39 years old presenting 73 cases (21\%). Conclusion: It was found that although the importance of nurses in this theme is well evidenced, as well as the high rates of suicides in Brazil, especially in the state of Pará, the number of studies that describe the relationship between nursing professionals and suicidal behavior is still low.
\end{abstract}

Keywords: Suicide; Mental health; Psychiatric nursing. 


\begin{abstract}
Resumen
Introducción: La ideación suicida es un elemento fundamental de un proceso denominado conducta suicida, que incluye: la planificación, el intento y el suicidio en sí. Es un camino que va desde la motivación y la ideación suicida hasta la planificación del método y la autoagresión. Brasil ocupa el undécimo lugar en el ranking mundial de suicidios. Objetivo: identificar el perfil epidemiológico de los suicidios notificados en el estado de Pará en el año 2019. Materiales y Métodos: Estudio epidemiológico descriptivo, a partir de datos de Suicidios ocurridos en el Estado de Pará en el año 2019, registrados en el Sistema de Información de Mortalidad y puestos a disposición por el Departamento de Informática del Sistema Único de Salud. Resultados: En 2019, se registraron 348 suicidios en el Estado de Pará, siendo los municipios de Belém (55 casos), Santarém (18 casos) e Itaituba (18 casos), las ciudades con mayor ocurrencia; en cuanto a la variable género, hubo una prevalencia del género masculino, con 272 (78,16\%); En cuanto a la franja de edad, 88 casos $(25,3 \%)$ tenían entre 20 y 29 años, seguidos por la franja de edad entre 30 y 39 años, que presentó 73 casos (21\%) y en cuanto al estado civil, los casos aislados representaron el 62,2\% (218) de los casos. Conclusión: A pesar de la importancia de las enfermeras en esta área, y de las altas tasas de suicidio en Brasil, particularmente en el estado de Pará, el número de estudios que describen la relación entre los profesionales de enfermería y el comportamiento suicida es todavía bajo.
\end{abstract}

Palabras clave: Suicidio; Salud mental; Enfermería psiquiátrica.

\title{
1. Introdução
}

A ideação suicida é um elemento fundamental de um processo chamado comportamento suicida, o qual inclui: planejamento, tentativa e o suicídio propriamente dito (Veloso et al, 2020, Santos et al, 2017).

Segundo Oliveira, et al. (2016), o suicídio é definido biopsicologicamente e influenciado por aspectos socioculturais que envolvem o indivíduo. Trata-se de um processo que vai desde a motivação e ideação suicida até o planejamento e a autoagressão.

Para a sociedade atual, o suicídio é uma das principais causas de morte, segundo dados do primeiro Relatório Global para prevenção do Suicídio, estima-se que 800 mil pessoas por ano se suicidem no mundo, uma a cada 40 segundos, tornandose um importante problema de Saúde Pública (Silva et al, 2016).

Conforme Gonçalves et al. (2020), cerca de um milhão de pessoas morrem por suicídio todos os anos, sendo a segunda maior causa de morte em jovens de 15 a 29 anos (Gonçalves et al., 2020; Veloso et al., 2020). O Brasil ocupa a $11^{\text {a }}$ posição no ranking mundial de suicídios onde nos anos de 2011 a 2016, foi-se registrado 48.204 casos de tentativas de suicídio, as quais ocorreram em maior número na região Sudeste e Sul do país (Silva et al, 2016, Claumann et al, 2018). No Brasil foram instituídas as Diretrizes Nacionais para Prevenção do Suicídio, por causa desse aumento, a fïm de ressaltar a importância de se falar sobre o tema, bem como promover a prevenção do suicídio (Oliveira et al, 2017).

Em um estudo comparativo entre Estados Unidos da América (EUA) e Alemanha, com 665 indivíduos de cada nação, em relação a ideações suicida e tentativas de suicídio, mostrou uma prevalência de 6,5\% de comportamento suicida nessa população em especifico (Santos et al, 2020). Em soma, um estudo com 15.927 universitários de 21 países evidenciou, em média, que $32 \%$ dos estudantes admitiram ter ideias suicidas (Barros, 2018).

Homens tendem a se suicidar mais, embora as tentativas de suicídio sejam maiores em mulheres, usando métodos mais letais como uso de armas de fogo, enquanto que mulheres utilizam método menos violento como intoxicações medicamentosas, envenenamento e afogamento (Erse et al, 2016; Raposo et al, 2016).

Cerca de $80 \%$ dos suicídios ocorre em países pouco desenvolvidos, estando o Brasil na oitava posição de números de suicídios nas Américas. Nesse contexto, o comportamento suicida é algo complexo e amplo mediante as suas intenções, assim como as suas consequências. Ele demostra a fragilidade que o sujeito se encontra, da mesma forma que reflete o quanto a vida está insuportável ao ponto de abdicar de todas as possibilidades que o futuro poderá vir a proporcionar, em prol do término do seu sofrimento propriamente dito (Vedana et al, 2019). 
Segundo Wanzinack et al. (2017), na visão de quem comete suicídio, ele é interpretado como o método para pôr fim ao sofrimento, desesperança, problemas pessoais e sociais. O comportamento suicida é bastante julgado por todos, sejam os próprios familiares, os amigos, ou mesmo a sociedade em si.

Segundo Pessoa et al. (2020), os fatores de risco mais relacionados ao suicídio são: término de relacionamento, violência sexual, depressão, transtorno de ansiedade, sentimentos de solidão, angústia e tristeza.

A depressão é a condição clinica mais estreitamente relacionada ao suicídio. Além disso, fatores sociodemográficos como desemprego, baixa escolaridade, estado civil e isolamento social, são fatores contribuintes no desenvolver de ideações suicidas (Raposo et al, 2016, Rossi et al, 2019).

O suicido é usado para apagar as angustias mentais na falta de outros meios psicológicos para defrontar os conflitos, fracassos e perdas. Visto isso, o comportamento suicida necessita ser encarado numa perspectiva abrangente à saúde mental, mediantes atitudes acolhedoras e abertura de um ambiente comunicativo interposto entre o sujeito em seu contexto sócio familiar e profissional, com intuito de estimular esperança e ofertar orientações (Martins et al, 2020).

Frente a esse problema, percebeu-se a necessidade de aprofundar o conhecimento sobre o assunto, com o objetivo de identificar perfil epidemiológico de suicídio notificado no estado do Pará no ano de 2019.

\section{Materiais e Métodos}

Trata-se de um estudo epidemiológico descritivo. Os dados foram obtidos por meio do Departamento de Informática do Sistema Único de Saúde - DATASUS, com abordagem descritiva do perfil epidemiológico de óbitos por suicídio no Estado do Pará, no ano de 2019, como referências para as bases populacionais e territoriais do Instituto Brasileiro de Geografia e Estatística (IBGE) no período estudado. Foram obtidas informações de pacientes na faixa etária de 5 a 80 anos ou mais, de ambos os sexos, utilizando-se as variáveis: faixa etária, gênero e estado civil.

Foi escolhido a abordagem descritiva pois a mesma analisa como a incidência (casos novos) ou a prevalência (casos existentes) de uma doença ou condição relacionada à saúde varia de acordo com determinadas características, como gênero, estado civil, idade, escolaridade e renda, entre outros fatores. Ou seja, esse método possibilita uma avaliação onde é possível avaliar as diferentes formas de uma doença ou condição, bem como suas ordens e classificações, possibilitando ao pesquisador uma melhor compreensão sobre os diversos fatores que possam influenciar uma determinada condição, doença ou fenômeno (Freire, 2018).

Além dos dados obtidos pelo DATASUS, documentos e portarias do Ministério da Saúde e da Organização Mundial da Saúde, também foram utilizados estudos sobre a temática. Diante disso, a pesquisa contou com uso das bases de dados: SciELO (Scientific Eletronic Library Online) e Biblioteca Virtual em Saúde (BVS), utilizando-se os descritores: Suicídio, Sistemas de Informação em Saúde, Monitoramento Epidemiológico, Saúde Mental e Enfermagem Psiquiátrica.

Foram excluídas dados que não corresponderam o período pré-estabelecido na pesquisa, como o ano de notificação e casos notificados em outros estados brasileiros.

\section{Resultados e Discussão}

A elevação dos indicadores mundiais de suicídio tem tornado esse tema uma problemática de repercussão global, dado os altos índices. No Brasil, durante o período de 2011 a 2018 foram notificados um total de 339.730 casos de violência autoprovocada (Brasil, 2019).

O presente estudo visa verificar o perfil epidemiológico de óbitos por suicídio no Estado do Pará, no ano de 2019, descrevendo os achados conforme as notificações por suicídio, onde foram analisadas as variáveis referentes às características 
sociodemográficas. Inúmeros fatores já foram identificados como predisponentes e precipitantes dos comportamentos suicidas, entre os quais: cidade, idade, sexo e situação conjugal são apontados.

Analisando a mortalidade por suicídio no estado do Pará, verificou-se que em 2019348 casos foram notificados ao Departamento de Informática do Sistema Único de Saúde - DATASUS, destaca-se que o município de Belém, capital do estado aparece em primeiro lugar com 15,8\% (55) casos de suicídio, seguido dos municípios de Santarém e Itaituba com 5,2\% cada (18) casos de suicídio, conforme evidenciado no (Quadro 1).

Quadro 1: Cidades no Estado do Pará e número de notificações por suicídio no ano de 2019 - Lesões autoprovocadas voluntariamente.

\begin{tabular}{|c|c|}
\hline MUNICÍPIO & ÓBITOS \\
\hline Abaetetuba & 2 \\
\hline Acará & 3 \\
\hline Afuá & 2 \\
\hline Alenquer & 1 \\
\hline Almeirim & 3 \\
\hline Altamira & 6 \\
\hline Anajás & 3 \\
\hline Ananindeua & 17 \\
\hline Anapu & 1 \\
\hline Bannach & 1 \\
\hline Barcarena & 6 \\
\hline Belém & 55 \\
\hline Bragança & 5 \\
\hline Brasil Novo & 2 \\
\hline Breu Branco & 1 \\
\hline Breves & 6 \\
\hline Bujaru & 3 \\
\hline Cametá & 7 \\
\hline Canaã dos Carajás & 1 \\
\hline Capitão Poço & 2 \\
\hline Castanhal & 9 \\
\hline Colares & 1 \\
\hline Conceição do Araguaia & 3 \\
\hline Concórdia do Pará & 1 \\
\hline Curionópolis & 1 \\
\hline Curralinho & 1 \\
\hline Curuá & 1 \\
\hline Curuçá & 2 \\
\hline Dom Eliseu & 1 \\
\hline Faro & 1 \\
\hline Floresta do Araguaia & 2 \\
\hline Garrafão do Norte & 1 \\
\hline Goianésia do Pará & 1 \\
\hline Gurupá & 1 \\
\hline Igarapé-Açu & 2 \\
\hline Igarapé-Miri & 2 \\
\hline Inhangapi & 2 \\
\hline
\end{tabular}




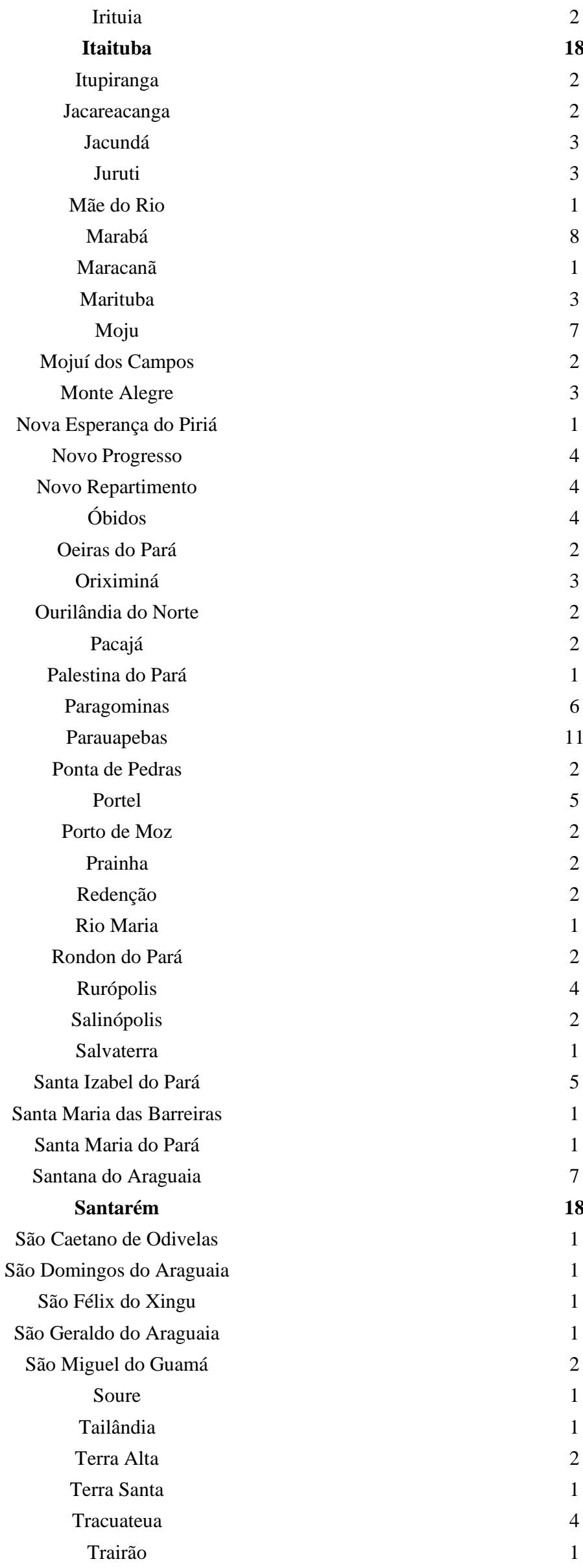




\begin{tabular}{ccc} 
& Tucumã & 2 \\
Tucuruí & 10 \\
Ulianópolis & Uruará & 2 \\
& Vitória do Xingu & 2 \\
& Xinguara & 2 \\
\hline TOTAL & & 5 \\
\hline
\end{tabular}

Fonte: MS/SVS/CGIAE - Sistema de Informações sobre Mortalidade - SIM.

Salienta-se que não se pode deixar de verificar as subnotificações e ausência de notificações para determinados casos de suicídio. Como exemplo, ocorrência de afogamentos em diversas regiões do Estado do Pará, subsistência de praias e rios, podem estar sob um campo de suicídio, o que pode impedir uma busca eficaz por esse achado (Batista, Araujo, Figueiredo, 2016).

Os dados epidemiológicos demonstram a crescente incidência das taxas de suicídio na população brasileira. As taxas oficiais de suicídio no País variaram entre 4 e 5 para cada 100 mil habitantes. No Brasil durante o período de 2011 a 2018 foram notificados 339.730 casos de violência autoprovocada, dos quais 154.279 (45,4\%) ocorreram na faixa etária de 15 a 29 anos, sendo 103.881 (67,3\%) nas mulheres e 50.388 (32,7\%) nos homens (Brasil, 2019).

Para facilitar a compreensão dos dados a serem apresentados, apresenta-se a partir de então, os números por região de Saúde no Estado do Pará. Relativo à variável gênero pode-se observar abaixo que 270 (77,59\%) eram do sexo masculino e 78 $(22,41 \%)$ do sexo feminino.

Quadro 2: Incidência de gênero de casos notificados de suicídio nas cidades do Estado do Pará no ano de 2019 - Lesões autoprovocadas voluntariamente.

\begin{tabular}{cccc}
\hline REGIÃO DE SAÚDE (CIR) & MASC & FEM & TOTAL \\
\hline Araguaia & 19 & 8 & 27 \\
Baixo Amazonas & 32 & 5 & 41 \\
Carajás & 21 & 6 & 27 \\
Lago de Tucuruí & 18 & 2 & 20 \\
Metropolitana I & 61 & 16 & 77 \\
Metropolitana II & 13 & 1 & 14 \\
Metropolitana III & 26 & 11 & 37 \\
Rio Caetés & 10 & 1 & 11 \\
Tapajós & 20 & 9 & 29 \\
Tocantins & 17 & 9 & 26 \\
Xingu & 14 & 3 & 17 \\
Marajó I & 6 & 1 & 7 \\
Marajó II & 9 & 6 & 15 \\
\hline TOTAL & $\mathbf{2 7 0}$ & $\mathbf{7 8}$ & $\mathbf{3 4 8}$ \\
\hline
\end{tabular}

Fonte: MS/SVS/CGIAE - Sistema de Informações sobre Mortalidade - SIM.

No estudo de Silva et al. (2017), verificou-se que a variável sexo manteve a associação mostrando que o sexo masculino referiram $78,16 \%$ suicida do que os do sexo feminino com $21,84 \%$. Todavia, esse dado corrobora com a literatura sobre os casos 
notificados de suicídio, que indica que as mulheres estão, de maneira geral, favorável à ideação suicida e os homens ao suicídio são que realmente executando o ato de suicídio (Quadro 2).

A maior parte dos dados demográficos encontrados na literatura demonstram que a tentativa de suicídio é mais comum em mulheres, mas o índice de suicídio executado é maior em homens, a cada três homens que falecem por suicídio uma mulher morre pela mesma causa. Percebe-se que os homens possuem dificuldade para procurar e aceitar que precisam de ajuda, mesmo encontrando-se em uma série de problemas emocionais ou psicológicos não costumam abrir-se, pedir o auxílio de alguém. E também utilizam métodos mais agressivos, o que reduz as probabilidades de sobrevivência (Silva, Motta, 2017).

No que se refere, o suicídio no sexo masculino, situações de ordem financeira podem ser mais complexas para os homens encararem tornando-se um fator estressante que também pode influenciar na conduta suicida. Visto que a cultura responsabiliza o homem em relação ao que se refere ao sustento da casa, quando há uma situação de crise financeira, o mesmo pode abalar-se extremamente, por carregar tal responsabilidade (Schlösser, Rosa, 2014).

Ressalta-se que o número de suicídios é considerado três vezes maior na população masculina do que na população feminina em países desenvolvidos, enquanto que para países em desenvolvimento essa relação se apresenta 50\% menor (Oms, 2018).

Autores como Sörberg et al. (2018), destacam que nessa conjuntura existem situações que fazem compreender melhor porque as altas taxas de suicídio entre os homens são maiores, dentre alguns motivos encontrados estão descritos para o impacto de questões socioeconômicas, o desempenho escolar, a resistência em buscar ajuda psiquiátrica, o efeito do divórcio e a prevalência de alcoolismo.

De modo geral, os dados mostram que o principal contribuinte de aumento do risco nos dois sexos é a idade. Estudos nacionais e internacionais demonstram que a mortalidade por causas externas predomina nos jovens do sexo masculino residentes de grandes cidades e em áreas com superposição de desvantagens sociais, sendo necessário pautar indicadores como violência comunitária e criminal, constância de tráfico de drogas e outras atividades ilegais como fatores importantes para o entendimento dos diferenciais entre os sexos segundo as diferentes faixas etárias (Oms, 2018).

O estudo revelou que no ano de 2019 a maioria dos suicídios ocorreu entre indivíduos de 20 a 29 anos de idade, com 88 casos ou seja $(25,3 \%)$, dentro deste período ainda se observa que entre 30 a 39 anos ocorreram em 73 casos (21\%) quando relacionado a outras faixas etárias deste mesmo ano (Quadro 3). 
Quadro 3: Incidência de casos notificados de suicídio no Estado do Pará no ano de 2019 segundo a faixa etária.

\begin{tabular}{|c|c|c|c|c|c|c|c|c|c|c|c|c|}
\hline REGIÃO DE SAÚDE & $\begin{array}{c}5 \text { A } 9 \\
\text { ANOS }\end{array}$ & $\begin{array}{l}10 \text { A } 14 \\
\text { ANOS }\end{array}$ & $\begin{array}{c}15 \text { A } 19 \\
\text { ANOS }\end{array}$ & $\begin{array}{c}20 \text { A } 29 \\
\text { ANOS }\end{array}$ & $\begin{array}{c}30 \text { A } 39 \\
\text { ANOS }\end{array}$ & $\begin{array}{c}40 \text { A } 49 \\
\text { ANOS }\end{array}$ & $\begin{array}{c}50 \text { A } 59 \\
\text { ANOS }\end{array}$ & $\begin{array}{l}60 \text { A } 69 \\
\text { ANOS }\end{array}$ & $\begin{array}{c}70 \text { A } 79 \\
\text { ANOS }\end{array}$ & $\begin{array}{c}80 \text { ANOS E } \\
\text { MAIS }\end{array}$ & $\begin{array}{c}\text { IDADE } \\
\text { IGNORADA }\end{array}$ & TOTAL \\
\hline Araguaia & - & 1 & 4 & 6 & 5 & 6 & 2 & 1 & 1 & 1 & - & 27 \\
\hline Baixo Amazonas & - & - & 5 & 15 & 6 & 3 & 6 & 6 & - & 1 & - & 42 \\
\hline Carajás & - & - & 1 & 7 & 12 & 3 & 2 & 1 & 3 & - & - & 29 \\
\hline Lago de Tucuruí & - & - & 4 & 5 & 1 & 2 & 3 & 4 & 1 & - & - & 20 \\
\hline Metropolitana I & - & 4 & 9 & 17 & 15 & 9 & 8 & 9 & 3 & - & 1 & 75 \\
\hline Metropolitana II & - & 2 & 4 & 2 & 3 & 3 & - & - & - & - & - & 14 \\
\hline Metropolitana III & - & 1 & 6 & 8 & 10 & 4 & 3 & 3 & 1 & - & - & 36 \\
\hline Rio Caetés & - & 1 & 2 & 1 & 4 & 2 & - & 1 & - & - & - & 11 \\
\hline Tapajós & - & - & 5 & 12 & 7 & - & 2 & 3 & - & - & - & 29 \\
\hline Tocantins & - & 2 & 4 & 4 & 5 & 7 & 2 & 1 & 1 & - & - & 26 \\
\hline Xingu & - & 2 & 3 & 7 & 1 & 2 & 1 & 1 & - & - & - & 17 \\
\hline Marajó I & - & - & - & 1 & 2 & 2 & - & 1 & - & - & - & 6 \\
\hline Marajó II & 1 & 2 & 4 & 3 & 2 & 1 & 1 & 1 & 1 & - & - & 16 \\
\hline TOTAL & 1 & 15 & 51 & 88 & 73 & 44 & 30 & 32 & 11 & 2 & 1 & 348 \\
\hline
\end{tabular}

Fonte: MS/SVS/CGIAE - Sistema de Informações sobre Mortalidade - SIM. 
No Brasil, a maior prevalência de casos notificados de lesão autoprovocada, assim como de tentativas de suicídio, se encontram na faixa etária entre os 20 e 49 anos. Fatores relacionados com o uso abusivo de álcool e outras drogas, presença de sofrimento psíquico, vulnerabilidade financeira e social atuam como fatores de risco importantes no cenário brasileiro (Brasil, 2017).

O suicídio entre os jovens aumentou em todo o mundo nos últimos anos, representando a $2^{\mathrm{a}}$ principal causa de morte nessa faixa etária. Os comportamentos de cunho suicida entre os homens jovens envolvem diversas motivações complexas, incluindo humor depressivo, abuso de substâncias, problemas emocionais, familiares e sociais, história familiar de transtorno psiquiátrico, rejeição familiar, negligência, além de abuso físico e sexual na infância (Marcola, Silva, 2019).

Com relação ao estado civil, aplicando-se as estimativas censitárias às variáveis solteiro, casado, divorciado e viúvo, no período estudado, o suicídio ocorreu com maior prevalência em pessoas solteiras, representando $62,2 \%$ (218) dos casos, logo atrás os casados com 15,8\% (55), conforme descreve o (Quadro 4).

Quadro 4: Incidência de casos notificados de suicídio nas cidades do Estado do Pará no ano de 2019 segundo estado civil.

\begin{tabular}{|c|c|c|c|c|c|c|c|}
\hline REGIÃO DE SAÚDE & SOLTEIRO & CASADO & VIÚVO & $\begin{array}{c}\text { SEPARADO } \\
\text { JUDICIALMENTE }\end{array}$ & OUTRO & IGNORADO & TOTAL \\
\hline Araguaia & 21 & 4 & 1 & - & 1 & - & 27 \\
\hline Baixo Amazonas & 28 & 6 & 1 & - & 7 & - & 42 \\
\hline Carajás & 16 & 9 & - & - & 3 & 1 & 29 \\
\hline Lago de Tucuruí & 10 & 8 & - & - & 1 & 1 & 20 \\
\hline Metropolitana I & 50 & 9 & 1 & 5 & 7 & 3 & 75 \\
\hline Metropolitana II & 8 & 3 & - & - & 2 & 1 & 14 \\
\hline Metropolitana III & 22 & 7 & 2 & 1 & 2 & 2 & 36 \\
\hline Rio Caetés & 9 & - & - & - & 1 & 1 & 11 \\
\hline Tapajós & 18 & 2 & - & 2 & 6 & 1 & 29 \\
\hline Tocantins & 12 & 2 & 1 & 1 & 9 & 1 & 26 \\
\hline Xingu & 11 & 3 & 1 & - & 1 & 1 & 17 \\
\hline Marajó I & 3 & - & - & - & 3 & - & 6 \\
\hline Marajó II & 10 & 2 & - & - & 4 & - & 16 \\
\hline TOTAL & 218 & 55 & 7 & 9 & 47 & 12 & 348 \\
\hline
\end{tabular}

Fonte: MS/SVS/CGIAE - Sistema de Informações sobre Mortalidade - SIM.

Ao se tratar de um evento que pode ser evitável, o suicídio a partir do momento que se consegue detectar o mais rápido possível, existe a possibilidade da intervenção adequada de imediato juntamente ao indivíduo. Contudo, as ações de prevenção ao suicídio no Brasil ainda são muito insipientes, ou seja, a realidade brasileira está baseada no predomínio da escassez de instrumentos operacionais que possam realmente ser capazes de lidar com essas questões referentes ao suicídio, em virtude disso, é notória a necessidade urgente de melhores práticas na criação e na implantação de programas governamentais de qualificação para os profissionais da saúde que atuam nesse contexto (Müller, Pereira, Zanon, 2017).

A sociedade apresentando a percepção sobre a importância da abordagem das questões relacionadas ao suicídio, no estudo sobre o assunto, Silva et al. (2017), explicam que a em consideração à gravidade do problema, na prática infelizmente o que se percebe é a baixa implementação de ações que abordem trabalhos de prevenção ao comportamento suicida, além de uma inabilidade perante a saúde mental e psiquiatria, bem como falta de recursos, e ausência de treinamentos e capacitações aos profissionais necessários. 
Suicídio é um ato intencional do indivíduo para extinguir a própria vida, em um estudo de Fernandes et al. (2020), estes descrevem que o suicídio vem aumentando em níveis preocupantes, fato que o torna uma importante questão para a saúde pública. A conduta suicida traz uma inquietação, especialmente, entre os profissionais da área de saúde.

De acordo com Silva et al. (2020), a equipe de enfermagem é responsável pelos cuidados iniciais ao paciente que tenta suicídio, além disso, devido à essência de sua atividade, que é o cuidado, permanece por mais tempo em contato direto com o ser assistido. Ao lado destes pacientes, tal equipe possui potencialidade para auxiliar na prevenção de novas tentativas de suicídio. Dessa forma, a enfermagem reconhece o suicídio como um problema atual e emergente, correlacionam-no com características próprias do paciente, mas também compreendem que fatores sociais e familiares influenciam na conduta suicida.

\subsection{Causas de suicídios}

De acordo com a Associação Brasileira de Psiquiatria (2014), existem alguns fatores que contribuem para o aumento no número de suicídios em todo mundo, destaca-se: tentativa previa de suicídio, doença mental, desesperança, desespero, desamparo, impulsividade, idade, gênero, dentre outros fatores. Os transtornos psiquiátricos mais comuns incluem depressão, transtorno bipolar, alcoolismo e abuso/dependência de outras drogas, transtornos de personalidade e esquizofrenia (Gomes et al, 2019).

A Campanha Setembro Amarelo foi criada com o intuito de levar ao conhecimento da população as formas de prevenção ao suicídio e alertá-la a respeito desse problema não só no Brasil, mas no mundo. Para que tal movimento informativo ocorra é possível se utilizar das informações gráficas e numéricas das taxas de suicídios ocorridos no país e como proceder em busca de orientação profissional caso seja necessário ajuda todas essas informações são disponibilizadas pelo Centro de Valorização da Vida (Bezerra, Silva, 2019).

Torna-se complexo um diagnóstico da doença por parte de um profissional, em decorrência desse transtorno aflorar-se e dar indícios somente no início da fase adulta. Tal transtorno muitas vezes está ligado a tentativas, ideação e suicídio consumado. Em conformidade com os estudos de Scussel et al. (2016), está claro que o Transtorno Bipolar tem sido bastante presente em indivíduos que tentam suicídio.

O preconceito e os aspectos socioculturais que permeiam o autoextermínio fazem com que os indivíduos que tentaram contra a própria vida e suas famílias tenham vergonha e medo de assumir o ato, gerando registros epidemiológicos não condizentes com a realidade (Silva, Motta 2017).

Outro fator bastante associado às tentativas de suicídio está relacionado ao uso de substâncias psicoativas, na literatura pertinente a essa linha de pesquisa, foram encontrados diversos artigos nos quais pode se observar uma relação forte entre o comportamento suicida. A isso se amplia o fato de que quando há a presença de transtorno depressivo, o risco de intento de suicídio é potencializado (Bezerra, Silva, 2019).

Cantão e Botti (2016), abordam o comportamento suicida em dependentes químicos, abrangendo drogas lícitas e ilícitas, os resultados das pesquisas dos mesmos corroboram o que foi trazido por outros autores, ressaltando-se a prevalência alta de suicídio em dependentes químicos e o quão é importante avaliar o risco de suicídio ao lidar com um paciente com dependência de substâncias psicoativas, sendo um fator que exige atenção no manejo com tais pacientes.

\subsection{Atendimento de enfermagem ao indivíduo pós tentativa de suicídio}

O profissional de enfermagem costuma ser o primeiro contato do paciente que tenta tirar o autocídio, seja na atenção primária ou na atenção terciária. Assim uma adequada avaliação e manejo desse sujeito é fundamental para prevenir novos comportamentos suicidas. Além disso, a enfermagem tem importante papel na assistência ao adolescente, pois, na atenção 
primária, deve-se realizar atividades de promoção da saúde voltadas a esta população com vistas a prevenir danos que possam repercutir na vida do sujeito e da comunidade (Gomes et al, 2019).

As práticas de cuidados realizados pelo profissional de enfermagem, sejam elas individuais ou atos coletivos, envolvem a abordagem clínica do processo de saúde física e emocional, e através da conjugação dos diferentes saberes implicados na produção de saúde deve ser tratada de forma interdisciplinar. Através da relação entre o enfermeiro e o paciente, se produzem falas, escutas, interpretações, bem como o sentimento de acolhimento pelo paciente (Gonçalves et al, 2015).

Ao deparar-se com um paciente com comportamento suicida, primeiramente, em relação a conduta do profissional, é preciso que o mesmo auxilie o indivíduo afim de que ele sinta-se em segurança, onde quer que se encontre. Para intervenções psicoterápicas em situações de crise, o terapeuta precisa conhecer profundamente as teorias que embasam esse tipo de psicoterapia e possuir alguns traços tais como: saber compreender o paciente, agir com naturalidade, tranquilidade e transmitir confiança (Silva, Motta, 2017).

A equipe de enfermagem executa ações que visam a atenção total dos que necessitam desse atendimento. Nesse sentido, os profissionais de enfermagem têm papel muito importante no acolhimento dessas pessoas, trazem segurança para os pacientes e principalmente para a família que fica desolada nesses momentos. Os profissionais enfermeiros ativos no serviço de saúde devem estar preparados e qualificados para identificar as características que o paciente com potencial suicida apresenta; observar a presença de pensamentos e atitudes que demonstrem desesperança, aflição e desamparo. O paciente com estas características devem ser abordados de forma clara e cautelosa, mantendo a serenidade, a empatia e abstendo-se das atitudes julgadoras (Marçal, Gonçalves, 2020).

O enfermeiro ao planejar ações preventivas voltadas ao suicídio, acolhendo as pessoas com uma escuta qualificada que permite avaliar o grau de risco e tomar as devidas condutas, contribui para eficácia na prevenção do suicídio. Estudos evidenciam que mais de 75\% das pessoas vítimas de suicídio buscaram um serviço de saúde no ano em que se deu a consumação do suicídio. Isto mostra o quanto é importante o enfermeiro está treinado para atuar de forma efetiva na detecção de comportamento suicida e acionar a redes de cuidados junto à família. É necessário compromisso e articulações do setor saúde nesta luta, a fim de reduzir os casos (Gomes et al., 2019).

\subsection{A importância da enfermagem no sistema de notificação}

O estudo de Sousa et al. (2019), identificou que, por falta de capacitação, alguns profissionais da saúde entendem o ato de tirar a vida como uma afronta aos serviços de saúde. É de suma importância que os profissionais da saúde tenham treinamentos sobre questões de suicídio para oferecer um suporte e acolhimento de forma adequada e ofertar estratégias capazes de prevenir o suicídio. Assim, por ser um problema de saúde pública, é necessário que os profissionais enfermeiros tenham interesse em procurar adquirir conhecimento, capacitação e cobrar dos órgãos gestores atualizações necessárias para que saibam atuar diante de casos de tentativa de suicídio, com o intuito de contribuir com a diminuição destes casos e encorajando o paciente sobre a importância da valorização da vida

O profissional de enfermagem, membro de uma rede multiprofissional que engloba cuidados de saúde nos setores em que a pessoa esteja inserida, deve prestar boa assistência, acolhimento, escuta, acionar fontes de apoio, verificar o tratamento adequado ao quadro clínico e preencher a ficha de notificação compulsória. Tais medidas de prevenção e recuperação adotadas pelas equipes multiprofissionais contribuem para a redução da ocorrência de suicídio. Desta forma, identificar e intervir adequadamente perante as pessoas com comportamento suicida é primordial para prevenir os suicídios. Com isso, vale compreender que a Organização Mundial da Saúde explica o comportamento suicida como ideação suicida, planejamento suicida, tentativa e consumação do suicídio em si (Marçal, Gonçalves, 2020). 


\section{Considerações Finais}

Considerando os dados epidemiológicos, destacando assim, que a atuação do enfermeiro é relevante na luta contra o suicídio, é importante salientar que a equipe de enfermagem na qual está diretamente ligada ao paciente, tenha uma visão holística e preparada com o apoio familiar para a detecção, atenção e humanização priorizada.

Portanto, devem-se refletir os desafios que o profissional enfermeiro deve enfrentar ao vivenciar situação da abordagem sobre a tentativa de suicídio e a assistência que deve ser prestada ao paciente e sua família.

Evidenciou-se no presente estudo, que apesar de ser bem evidenciado a importância do enfermeiro nessa temática, bem como os elevados índices de suicídios ocorridos no Brasil em foco no estado do Pará, ainda é baixo o número de pesquisas que descrevem a relação entre o profissional de enfermagem frente ao comportamento suicida. é necessário maior enfoque à problematização dentro da atenção primária como promoção da prevenção do suicídio, visando uma linguagem de fácil interpretação, com direcionamento contínuo que englobe toda a equipe multidisciplinar.

Vale ressaltar que a diferença entre os pacientes com tentativas de suicídio e o suicídio em si, estão relacionados com faixa etária, gênero e estado civil, além do uso de substâncias psicoativas, nível de escolaridade e classe social.

Dessa forma faz-se necessário enfatizar que as autoridades públicas tem o dever de oferecer todo suporte necessário a estes pacientes, bem como a criação e implementação de políticas públicas de saúde e programas voltados para o tratamento e prevenção dos grupos mais suscetíveis, evitando assim que milhares de indivíduos cometam suicídio. Acredita-se ainda que seria de grande relevância que a temática voltada para o suicídio, bem como seus fatores de risco fossem estudados e aprofundados posteriormente, com o objetivo de agregar mais conhecimento e promover novas descobertas afim de proporcionar melhores condições de saúde, interpretação da doença aos pacientes e seus familiares.

\section{Referências}

Batista, N. O., Araujo, J. R. C. \& Figueiredo, P. H. M (2016). Incidência e perfil epidemiológico de suicídios em crianças e adolescentes ocorridos no Estado do Pará, Brasil, no período de 2010 a 2013. Rev Pan-Amaz Saude, 7(4), 61-66. http://dx.doi.org/10.5123/s2176-62232016000400008.

Bezerra, J. J. \& Silva, F. V (2019). As cores da vida: Estratégias biopolíticas nas campanhas setembro amarelo, outubro rosa e novembro azul. Revista Eletrônica do Netlli, 8(2), 728-741. DOI:10.47295/mgren.v8i2.1902.

Brasil, Ministério da Saúde. (2019) Secretária de Vigilância em Saúde. Perfil epidemiológico dos casos notificados de violência autoprovocada e óbitos por suicídio entre jovens de 15 a 29 anos no Brasil, 2011 a 2018. Brasília (DF): Ministério da Saúde; 50(24), 1-14.

Claumann, G. S., Araújo, A.P., Santos, D.A.S. \& Pelegrini, A. (2018). Prevalência de pensamentos e comportamentos suicidas e associação com a insatisfação corporal em adolescentes. J. bras. psiquiatr., 67(1), 3-9. https://doi.org/10.1590/0047-2085000000177.

Cantão, L. \& Botti, N (2016). Comportamento suicida entre dependentes químicos. Revista Brasileira de Enfermagem, 69(2), 389-396. Doi: https://dx.doi.org/10.1590/0034-7167.2016690224i

Erse, M. P. Q. A. M. P., Simões, P., Maria, R., Façanha, N., Daniel, J., Alves, F.M., Amélia, L., Costa, E.L., Rosalinda, C., Sampaio, T.M., Pereira, M.E.S. \& Carlos, J. (2016). Depressão em adolescentes em meio escolar: Projeto + Contigo. Rev. Enf. Ref., Coimbra , 9, 37-45. https://www.redalyc.org/articulo.oa?id=388245833005.

Fernandes, F. Y., Freitas, B. H. B. M. D., Marcon, S. R., Arruda, V. L. D., Lima, N. V. P. D., Bortolini, J. \& Gaíva, M. A. M. (2020). Tendência de suicídio em adolescentes brasileiros entre 1997 e 2016. Epidemiologia e Serviços de Saúde, 29, e2020117. http://dx.doi.org/10.1590/s1679-49742020000400025.

Freire, M. C. M., \& Pasttussi, M. P. (2018). Princípios da Pesquisa. Tipos de estudo. Metodologia científica: ciência, ensino, pesquisa. Research, Society and Development, 9(12), e18391210953. DOI: http://dx.doi.org/10.33448/rsd-v9i12.18391210953.

Gonçalves, F. S., Freitas, J. L., Albino, M. B. \& Souza, O. N. F. R. (2020). Aspectos socioeconômicos e risco de suicídio em adolescentes fluminenses. Braz. J. of Develop., 6(11), 92263-92283. DOI:10.34117/bjdv6n11-634. DOI:10.34117/bjdv6n11-634.

Gomes, E. R., Iglesias, A. \& Constantinidis, T. C. (2019). Revisão Integrativa de Produções Científicas da Psicologia Sobre Comportamento Suicida. Revista Psicologia e Saúde, 11 (2), 35-53. 10.20435/pssa.v11i2.61

Martins, S. G., Pereira, A. L. R. R., Costa, S. M. A. da, Reis, S. M. dos, Simor, A., Santana, M. E. de. \& Martins, A. C. G. S. Epidemiological and clinical profile of patients with attempted suicide seen at a psychiatric referral hospital. Research, Society and Development, 9(12), e18391210953. DOI: 10.33448/rsdv9i12.10953. 
Marçal, S. R., \& Gonçalves, J. R. (2020). Estratégias de intervenção do enfermeiro diante do comportamento e tentativa de autoextermínio. Revista JRG de Estudos Acadêmicos, 3(6), 56-68. https://doi.org/10.5281/zenodo.4292340.

Marcolan, J. F. \& Silva, D. A. (2019). O comportamento suicida na realidade brasileira: aspectos epidemiológicos e da política de prevenção. Revista M, 4 (7), 31-44. https://doi.org/10.9789/2525-3050.2019.v4i7.31-44.

Oliveira, C. T. de, Collares, L. A, Noal, M. H. O. \& Dias, A. C. G. Percepções de uma equipe de saúde mental sobre o comportamento suicida. Gerais: revista interinstitucional de psicologia, 9 (1), 78-89. http://hdl.handle.net/10183/163633.

Pessoa, D. M. S., Freitas, R. J. M., Melo, J. A. L., Barreto, F. A., Melo, K. C. O., Dias, E. C. S. (2020). Assistência de Enfermagem na Atenção Primária à Saúde de adolescentes com ideações suicidas. REME - Rev Min Enferm, 2, e-1290. http://www.dx.doi.org/10.5935/1415-2762.20200019

Rossi, L. M., Quevedo, T. M., Speranza, M. \& Barboza, M. F. C. (2019). Crise e saúde mental na adolescência: a história sob a ótica de quem vive. Cad. Saúde Pública, Rio de Janeiro, 35(3), e00125018. https://doi.org/10.1590/0102-311X00125018.

Raposo, J. V., Soares, A. R., Silva, F., Fernandes, M. G. \& Teixeira, C. M. (2016). Níveis de ideação suicida em jovens adultos. Estud. psicol., 33(2), 345-354. https://doi.org/10.1590/1982-02752016000200016.

Santos, H. G. B., Reschetti, S. M., Martínez, M. E., Nunes, M. B. \& Cabral, P. M. P. (2017). Fatores associados à presença de ideação suicida entre universitários. Rev. Latino-Am. Enfermagem, 25, e2878. https://doi.org/10.1590/1518-8345.1592.2878.

Santos, H. G. B., Reschetti, S. M., Martínez, M. E., \& Nunes, M. B. (2019). Ideação suicida em estudantes universitários: um perfil sociodemográfico. Psicologia Argumento,36 (92), 237-253. ISSN 1980-5942.

Silva, W. dos S., Ulisses, S. M., Malheiros, T. da C., Gonçalves, F. M. \& Pinho, D. F. de M. (2016). A influência de fatores de risco e proteção frente à ideação suicida. Psicologia, Saúde e Doenças [Internet], 17(3), 515-526. https://www.redalyc.org/articulo.oa?id=36249164016.

Silva, J. V. S.; \& Motta, H. L. (2017). Comportamento suicida: uma revisão integrativa da literatura. Educação, Psicologia e Interfaces, 1(2), 51-67. https://doi.org/10.37444/issn-2594-5343.v1i2.25.

Silva, L. D. L. T., Vecchia, B. P., Ramos, T. M., \& Costa, T. A. F. (2020). Profissionais de enfermagem de um serviço de urgência e emergência frente ao suicídio na adolescência. Revista Eletrônica Acervo Saúde, 12(10), e4042-e4042. https://doi.org/10.25248/reas.e4042.2020.

SILVA, K. F. A., MARIANY, A. A. \& DANIELA, P.C. (2016). Suicídio: Uma escolha existencial frente ao desespero humano. Pretextos-Revista da Graduação em Psicologia da PUC Minas [Internet], 184- 203. http://periodicos.pucminas.br/index.php/pretextos/article/view/13618.

Sousa, J. F., De Carvalho Sousa, V., De Carvalho, C. M. S., Amorim, F. C. M., Fernandes, M. A., Coelho, M. C. V. S., \& Soares, J. (2019). Prevenção ao suicídio na atenção básica: concepção de enfermeiros. Revista Cuidarte, 10(2), 4. https://doi.org/10.15649/cuidarte.v10i2.609.

SCUSSEL, F., Cechinel, L. S., Soares, L. B. \& Feier, G. (2016). Perfil clínico dos pacientes com transtorno bipolar atendidos em um ambulatório especializado na região sul catarinense. Arq. Catarin Med. out-dez; 45(4), 03-10. http://www.acm.org.br/acm/seer/index.php/arquivos/article/view/133.

Schlösser, A., Rosa, G. \& More, C. Revisão: comportamento suicida ao longo do ciclo vital. Temas em Psicologia, 22(1), 133-145, 2014. Doi: https://dx.doi.org/10.9788/TP2014.1-11

Vasconcelos, P. J. de A., Silveira, R. M., Moreira, F. J. O. J. \& Ludermir, A. B. (2020). Tentativa de suicídio, transtorno de estresse pós-traumático e fatores associados em mulheres do Recife. Rev. bras. epidemiol, 23, e200010. https://doi.org/10.1590/1980-549720200010.

Veloso, L. U. P., Soares, C. L. L., Carvalho, J. S. S., Ferreira, C. S. M., Martins, A. S. G. \& Guedes, F. J. S. J. (2019). Ideação suicida em universitários da área da saúde: prevalência e fatores associados. Rev. Gaúcha Enferm., 40, e20180144. https://doi.org/10.1590/1983-1447.2019.20180144.

Vedana, K. G. G. \& Zanetti, A. C. G. (2019). Atitudes de estudantes de enfermagem relacionadas ao comportamento suicida. Rev. Latino-Am. Enfermagem, 27, e3116. https://doi.org/10.1590/1518-8345.2842.3116.

Wanzinack, C., Temoteo, A. \& Oliveira, A. L. de. (2017). Mortalidade por suicídio entre adolescentes/jovens brasileiros: um estudo com dados secundários entre os anos de 2011 a 2015. Revista Eletrônica Interdisciplinar, 10(2), 106-117. 